\title{
Targeted expression of a dominant-negative EGF-R in the kidney reduces tubulo-interstitial lesions after renal injury
}

\author{
Fabiola Terzi, ${ }^{1}$ Martine Burtin, ${ }^{1}$ Mehrak Hekmati, ${ }^{1}$ Pierre Federici, ${ }^{2}$ \\ Giselle Grimber, ${ }^{2}$ Pascale Briand, ${ }^{2}$ and Gérard Friedlander ${ }^{1}$ \\ ${ }^{1}$ Institut National de la Santé et de la Recherche Médicale (INSERM) Unité 426 and Department of Physiology, \\ Faculté de Médecine Xavier Bichat, Université Paris 7, Paris, France \\ ${ }^{2}$ INSERM Unité 380, Institut Cochin de Génétique Moléculaire, Paris, France
}

Address correspondence to: Fabiola Terzi, INSERM Unité 426, Faculté Xavier Bichat BP416, 16 Rue Henri Huchard 75870 Paris, Cedex 18, France. Phone: 33-1-44-85-62-70; Fax: 33-1-42-28-15-64; E-mail: terzi@cochin.inserm.fr.

Received for publication September 1, 1999, and accepted in revised form May 24, 2000.

\begin{abstract}
The role of EGF in the evolution of renal lesions after injury is still controversial. To determine whether the EGF expression is beneficial or detrimental, we generated transgenic mice expressing a $\mathrm{COOH}$-terminal-truncated EGF-R under the control of the kidney-specific type $1 \gamma$-glutamyl transpeptidase promoter. As expected, the transgene was expressed exclusively at the basolateral membrane of proximal tubular cells. Under basal conditions, transgenic mice showed normal renal morphology and function. Infusion of EGF to transgenic animals revealed that the mutant receptor behaved in a dominantnegative manner and prevented EGF-signaled EGF-R autophosphorylation. We next evaluated the impact of transgene expression on the development of renal lesions in two models of renal injury. After $75 \%$ reduction of renal mass, tubular dilations were less severe in transgenic mice than in wild-type animals. After prolonged renal ischemia, tubular atrophy and interstitial fibrosis were reduced in transgenic mice as compared with wild-type mice. The beneficial effect of the transgene included a reduction of tubular cell proliferation, interstitial collagen accumulation, and mononuclear cell infiltration. In conclusion, functional inactivation of the EGF-R in renal proximal tubular cells reduced tubulo-interstitial lesions after renal injury. These data suggest that blocking the EGF pathway may be a therapeutic strategy to reduce the progression of chronic renal failure.
\end{abstract}

J. Clin. Invest. 106:225-234 (2000).

\section{Introduction}

EGF-R and its ligands are believed to play a pivotal role in renal development and pathology $(1,2)$. EGF-R binds and is activated by a family of growth factors, comprised of EGF itself, TGF- $\alpha$, heparin-binding EGF-like growth factor (HB-EGF), amphiregulin, epiregulin, and betacellulin (3). Some of these factors are secreted by renal tubular cells (4), and EGF-R is abundantly expressed along the nephron (5), suggesting that EGF pathway acts as an autocrine/paracrine system in the kidney.

In vitro, addition of EGF-R ligands to renal cells promotes several responses, including cell proliferation (6, $7)$, cell migration $(8,9)$, collagen production $(10$,$) and$ tubulogenesis (11). In vivo, there is increasing evidence for the role of EGF-R pathway in several renal pathological conditions characterized by intense cell proliferation, such as acute tubular necrosis, nephron reduction, polycystic kidney disease, or adenocarcinoma $(1,2)$. Indeed, it has been shown that: (a) in the regenerating phase after ischemic or toxic renal injury, infusion of EGF enhanced recovery from acute tubular necrosis (12, 13); (b) immunostainable EGF increased in kidney from day 7 to several months after nephron reduction (14-18); (c) large amounts of EGF, associated with increased activity and apical mislocalization of EGF-R, were detect- ed in cystic-lining epithelial cells in human patients and in several mouse models of autosomal recessive polycystic kidney disease $(19,20)$; (d) both the ligands (EGF, TGF- $\alpha$ ) and the EGF-R itself were overexpressed in human renal cell carcinoma, and their levels correlated with the rapidity of cancer evolution (21). Furthermore, in three different models of transgenic mice expressing, respectively, genes encoding for TGF- $\alpha$ (22), for c-erb-B2 (23), an integral membrane protein similar to EGF-R, or for T24-ras (24), a protein involved in the transduction of EGF-R, activation of EGF-R pathway resulted in tubular hyperplasia and development of renal cysts.

On the other hand, experimental approaches aiming to block EGF-R signaling seems to ameliorate the evolution of renal diseases. Richard et al. (25) used a genetic approach (by breeding mice that bear the orpk cystic mutation and the waved-2 EGF-R mutation, respectively) to demonstrate that decreasing activation of the EGF-R diminished collecting tubular cyst formation in mice. In our laboratory, we showed that a moderate restriction of dietary sodium content, which reduced the progression of tubulo-interstitial lesions after nephron reduction, blunted the upregulation of EGFAP1 cascade and the tubular hyperplasia in remnant kidney in subtotally nephrectomized rats (18). Finally, 
injection of a mouse-human chimeric anti-EGF-R antibody inhibited the growth of human renal-cell carcinoma xenografts in nude mice (26). Together these results strongly suggest that EGF-R is a major determinant in the development of renal lesions, possibly through an increase of tubular cell proliferation.

Genetic approaches aiming to bring direct evidence for a role of EGF-R activation in renal physiopathology face two major difficulties: EGF-R-null mice are not viable postnatally (27-29), and inactivation of one of the numerous EGF-R's ligands could not be informative because of a considerable redundancy in the ligands, as suggested by TGF- $\alpha$-inactivation studies (30). For these reasons, we developed an EGF-R dominantnegative strategy, based on targeted expression of a $\mathrm{COOH}$-terminal-truncated receptor (HERCD-533) in the kidney by using the $5^{\prime}$ regulatory region of $\gamma$-glutamyl transpeptidase type 1 ( $\gamma G T-1)$ gene. We chose this promoter in order to target proximal tubular cells, which are the major site of EGF binding in the kidney (5) and to avoid renal developmental malformations ( $\gamma$ GT-1 gene is mainly expressed from 3 weeks after birth; ref. 31). We next investigated the impact of transgene expression on the development of renal lesions in two different models of renal injury: subtotal nephrectomy and prolonged renal ischemia.

We found that, under control conditions, transgenic mice had normal renal morphology and renal function. After renal injury, however, homozygous transgenic mice were resistant to the development of renal lesions. These findings demonstrated the crucial role of EGF-R signaling in renal deterioration process and proved the potential for a therapeutic application of EGF-R mutants in the treatment of progression of chronic renal failure.

\section{Methods}

Plasmid construction and generation of transgenic mice. To create the $\gamma$ GT-EGF-R-M fusion gene, the $\gamma \mathrm{GT}$ promoter was removed from the plasmid pG2.2rasT24 (kindly provided by M.W. Lieberman, Department of Pathology, Baylor College of Medicine, Houston, Texas, USA) and cloned into the pBluescript plasmid, generating the plasmid $\gamma$ GT. The mutant EGF-R (HERCD533) was removed from the Prk5HerNA8 plasmid (kindly provided by A. Ullrich, Department of Molecular Biology, Max-Planck-Institut für Biochemie, Martinsried, Germany) and ligated into XbaI-digested $\gamma \mathrm{GT}$ plasmid. Finally, a $1.2-\mathrm{kb}$ fragment, containing the poly(A) sequence for the human globin gene, was isolated and cloned into the $\gamma$ GT-EGF-R-M plasmid. The transgene was excised from the $\gamma$ GT-EGF-R-M plasmid with $P v u \mathrm{I}$ and $\mathrm{XhoI}$ and microinjected into the pronucleus of fertilized one-cell C57BL/ $6 \times \mathrm{DAB}_{2} \mathrm{~F}_{1}$ mouse eggs. Transgenic mice were identified by Southern blot analysis using a ${ }^{32} \mathrm{P}$-labeled $\mathrm{XbaI} / \mathrm{XhoI}$ fragment, corresponding to the poly(A) of human globin gene. Line 8 , 20 , and 35 were established and maintained by breeding to $\mathrm{C} 57 \mathrm{BL} / 6 \times \mathrm{DAB}_{2} \mathrm{~F}_{1}$ hybrid animals.
All animal procedures were conducted in accordance with French government policies (Service Vétérinaire de la Santé et de la Production Animale, Ministère de l'Agriculture).

Experimental models of renal injury. All the experiments were performed on adult ( 2 to 3 months) homozygous transgenic mice (EGF-R-M) and on age- and sexmatched wild-type animals. Homozygous transgenic mice were obtained by homozygous EGF-R-M intercrosses, whereas wild-type mice were obtained by breeding $\mathrm{C} 57 \mathrm{BL} / 6 \times \mathrm{DAB} 2 \mathrm{~F}_{1}$ hybrid animals. The crosses were realized in parallel in the same animal facility.

Subtotal nephrectomy. Subtotal nephrectomy (Nx; 75\% excision of total renal mass) was performed as described previously (32) on 18 transgenic and 18 wild-type mice. Control mice ( $n=9$ in each group) were subjected to a sham operation with decapsulation of both kidneys.

Initially, the study was designed to be completed 2 months after surgery, at a time point when remnant kidney showed severe renal lesions, at least in rats (33). Since morphology of remnant kidneys revealed a wellpreserved renal tissue in both wild-type and transgenic mice at this time ( $n=5$ for each group), as well as at 6 months ( $n=5$ for each group), mice ( $n=8$ for each group) had to be sacrificed 8 months after surgery.

Prolonged renal ischemia. Prolonged renal ischemia (PRI; 50-minute clamping of left renal vascular pedicle) was performed as described previously (34). Mice were sacrificed 2, 4, 7, 14, and 28 days after surgery. At each time, six transgenic and six wild-type animals were examined. To determine the rate of cellular proliferation in the normal kidney, five normal nonoperated transgenic and wild-type mice were studied.

EGF-R phosphorylation. Adult ( 2 months) transgenic and wild-type mice were subjected to intracardiac injection of EGF ( $1 \mu \mathrm{g} / \mathrm{g}$ body weight) in isotonic saline. After 10 or 20 minutes, mice were sacrificed, and kidneys were removed and homogenized in a buffer containing $50 \mathrm{mM}$ Tris- $\mathrm{HCl}, \mathrm{pH}$ 8.0, 1 mM EDTA, 150 $\mathrm{mM} \mathrm{NaCl}, 1 \%$ Triton $\mathrm{X}-100,0.1 \% \mathrm{SDS}, 1 \mathrm{mM}$ sodium orthovanadate, $10 \mu \mathrm{g} / \mathrm{mL}$ pepstatin $\mathrm{A}, 10 \mu \mathrm{g} / \mathrm{mL}$ leupeptin, $2 \mu \mathrm{g} / \mathrm{mL}$ aprotinin, and $100 \mu \mathrm{g} / \mathrm{mL}$ PMSF. Kidneys from normal noninjected transgenic and wildtype mice were also prepared to evaluate the basal level of EGF-R phosphorylation.

Western blot analysis. To analyze the phosphorylation of EGF-R protein, equivalent amounts of proteins were immunoprecipitated with a rabbit polyclonal anti-EGF$\mathrm{R}$ antibody (Ab-22, kindly provided by E. Earp, Lineberger Cancer Center, University of North Carolina, Chapel Hill, North Carolina, USA), which recognizes murine or human EGF-R intracellular domain. The immunoprecipitates were resolved in 7\% SDS-polyacrylamide gels and transferred to a nitrocellulose membrane (Biorad, Ivry sur Seine, France). The membrane was incubated with a recombinant anti-phosphotyrosine antibody linked with horseradish peroxidase (RC20; Transduction Laboratories, Interchim, Montlucon, France), diluted 1:2,500. Immunoreactive proteins were detected by enhanced 
chemiluminescence (ECL; Amersham Pharmacia Biotech, Courtaboeuf, France). The films were scanned using a Scan-Jet 3c/ADF (Hewlett Packard, Palo Alto, California, USA) and the signals were quantified using the NIH image software (Bethesda, Maryland, USA).

To evaluate the expression of endogenous and of mutated EGF-R, proteins were extracted from cultured proximal tubular cells (35), resolved in 7\% SDSpolyacrylamide gels, and transferred to a nitrocellulose membrane. The membrane was incubated, first, with the mAb 108 antibody (kindly provided by A. Ullrich), which specifically recognizes the extracellular domain of the human EGF-R, diluted 1:2,000, then with the polyclonal Ab-22 (see above), diluted $1: 2,000$. Immunoreactive proteins were detected by an anti-mouse antibody and by an anti-rabbit antibody diluted 1:4,000, followed by ECL.

Northern blot analysis. Total RNA was extracted from kidneys using the RNAzol kit (Bioprobe, Montreuilsous-Bois, France). RNA was fractionated on a $1 \%$ agarose-formaldehyde gel and transferred onto nylon membrane (Zetabind; CUNO Inc., Meridien, Connecticut, USA). Prehybridization, hybridization, and washing were carried out according to the manufacturer's recommendations. RNA was quantified by Packard Instant Imager. The cDNA probes were labeled by random priming method (Amersham Pharmacia Biotech) using alpha ${ }^{32} \mathrm{P}-\mathrm{dCTP}$. The EGF-R-M mRNA was detected with the entire $1.2-\mathrm{kb}$ poly $(\mathrm{A})$ of human globin cDNA, as described in Southern blot. The $18 \mathrm{~S}$ and $28 \mathrm{~S}$ ethidium bromide staining were used in order to quantify the RNA loading on the gel and to compare the intensity of hybridization obtained in the different lanes.

${ }^{125}$ I-EGF-binding experiments. Proximal tubules were isolated from kidneys of transgenic and wild-type mice. Briefly, kidneys were removed, decapsulated, and dilacerated at $4^{\circ} \mathrm{C}$ by pressure (homogenizer Dounce no. 7; Poly Labs, Strasbourg, France) in HBSS. Proximal tubules were separated by filtering on a nylon mesh $(106 \mu \mathrm{m})$ and resuspended in PBS containing $0.1 \%$ BSA (PBS/BSA). Preliminary experiments were performed to determine the optimal binding conditions in terms of protein concentration and incubation time. Equivalent amounts of tubules (corresponding to $300 \mu \mathrm{g}$ of proteins) were incubated for 4 hours at $4{ }^{\circ} \mathrm{C}$ with increasing concentrations (from 0.01 to 10 $\mathrm{nM}$ ) of ${ }^{125}$ I-EGF (Amersham Pharmacia Biotech) with and without 1,000-fold excess of unlabeled EGF. After incubation, tubules were centrifuged, washed with $\mathrm{PBS} / \mathrm{BSA}$, and the radioactivity was measured in a gamma counter. The nonspecific binding was subtracted from the total counts at each concentration point to yield specific binding.

Renal morphology. Nonoperated kidneys from transgenic and sex- and age-matched wild-type mice, removed at different times after birth (1-18 months), as well as kidneys from mice that underwent different models of renal injury (see above), were fixed in $4 \%$ formalin overnight, ethanol dehydrated, and paraffin embedded.
Four-micrometer-thick sections were stained with periodic acid Schiff (PAS), with Masson's trichrome, and with Sirius red.

All sections were evaluated by a pathologist who was unaware of the group examined. A semiquantitative score was developed to evaluate the degree of damage after renal injury, according to established methodology (36).

After subtotal nephrectomy, the degree of damaged tubules was determined on PAS-stained sections by scoring 100 proximal tubules per kidney in randomly selected microscopic fields $(\times 400)$. Tubular lesions were graded from 0 to $3+$, according to the severity of tubular dilations.

After prolonged renal ischemia, the degree of tubulointerstitial injury (consisting of at least one of the following: tubular atrophy, interstitial fibrosis, interstitial cell infiltration) was determined on Masson's trichrome-stained sections, by scoring 20 randomly selected microscopic fields per kidney $(\times 400)$, using a $0-4+$ injury scale. Injury scale 0 means no tubulo-interstitial lesions, whereas $1,2,3$, and 4 correspond to $1-10$, $11-25,26-50$, and $51-100 \%$ of involvement of microscopic field, respectively. The degree of collagen accumulation was determined using the same scale on Sirius red-stained sections, by scoring 20 randomly selected microscopic fields per kidney $(\times 400)$.

The number of nuclei per tubular section was counted on PAS-stained sections. One-hundred proximal tubules per kidney were analyzed in randomly selected microscopic field $(\times 400)$.

Immunohistochemistry. Human EGF-R immunostaining was performed on frozen tissue sections. Fivemicrometer-thick sections were incubated for 1 hour at $4{ }^{\circ} \mathrm{C}$ with a mouse monoclonal anti-human EGF-R antibody (GR01; Calbiochem, Cambridge, United Kingdom), that specifically recognizes EGF-R extracellular domain, diluted $1: 10$, and then fixed in $4 \%$ icecold formalin at $4{ }^{\circ} \mathrm{C}$ for 5 minutes. Bound primary antibody was detected with biotinylated goat antimouse IgG (DAKO, Trappes, France), followed by avidin/biotin/peroxidase system (kit LSAB; DAKO). Then, 3-amino-9-ethylcarbazole (AEC; DAKO) was used as chromogen, and sections were counterstained with Meyer's hemalum and mounted in glycerol gelatin (Merck, Nogent-sur-Marne, France).

Negative controls were obtained by replacing specific antisera with nonimmune sera and by staining renal sections from wild-type mice with specific antisera.

Proliferating cell nuclear antigen (PCNA) immunostaining was performed on paraffin-embedded tissue sections, as described previously (34). The number of PCNA-labeled nuclei was determined in 20 randomly selected microscopic fields moving from the outer to the deep cortex $(\times 200)$.

Renal function. Renal function was evaluated in transgenic and wild-type mice at different times after birth, from 1 to 18 months. Urine samples were collected using Tecniplast metabolic cages over 24 hours and blood samples were obtained by cava puncture after urine collection. 

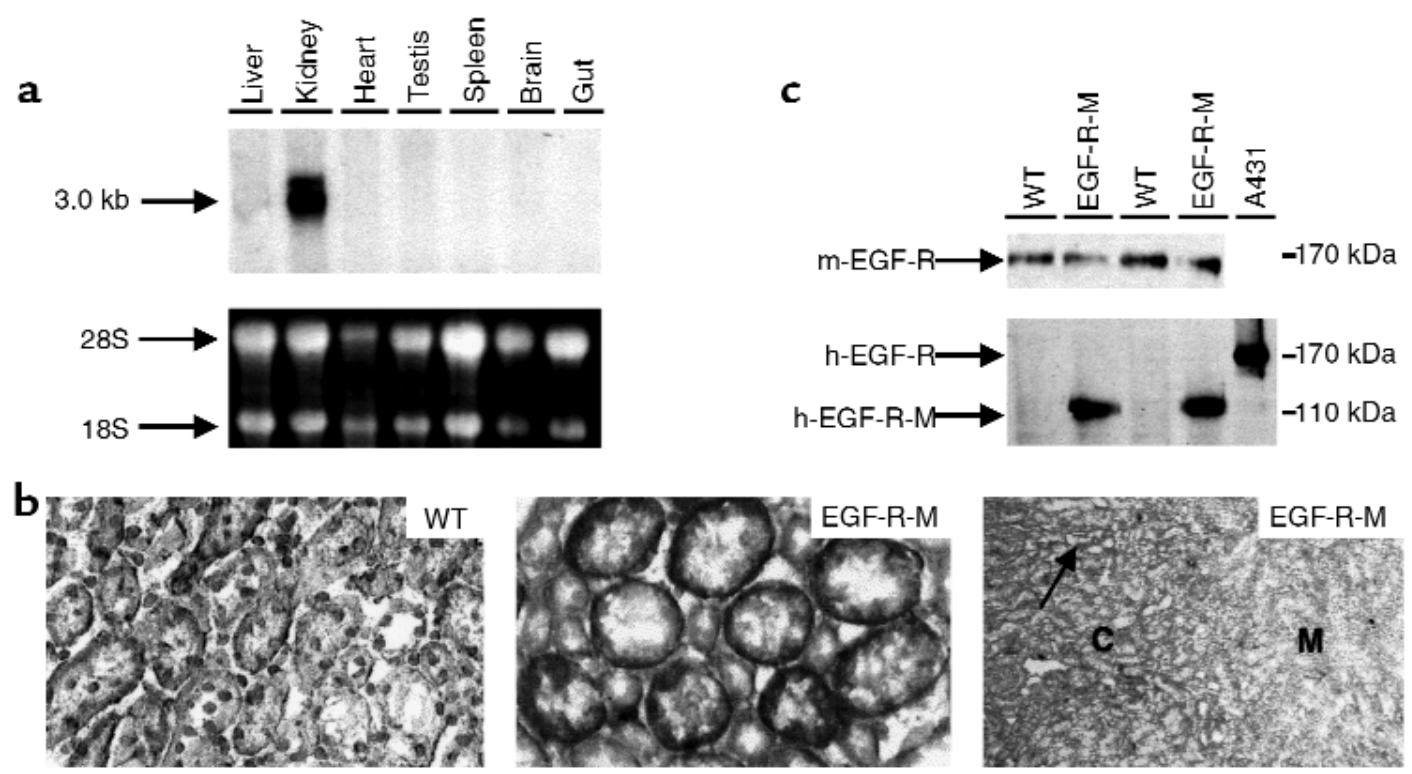

Figure 1

Transgene expression and function in transgenic mice. (a) Northern blot analysis. The transgene was detected exclusively in the kidney using the human $\beta$-globin poly(A) probe. (b) Immunohistochemical analysis with a specific anti-human EGF-R antibody. The transgene was localized in basolateral membranes of proximal transgenic tubules (EGF-R-M, middle panel), but not in those of wild-type membranes (WT, left panel). Specific staining was detected in the cortex (C) but not in the medulla $(M)$ of transgenic mice (right panel). (c) Western blot analysis of mouse (m-EGF-R) and human (h-EGF-R) EGF receptors in proximal tubular cells from WT and EGF-R-M mice. Similar amounts of endogenous EGF-R were detected by a specific anti-EGF-R intracellular domain antibody in EGF-R-M and WT mice ( $m$-EGF-R), whereas a $110-\mathrm{kDa}$ protein, corresponding to the truncated receptor, was evidenced in transgenic but not in wild-type mice, by a specific anti-human EGF-R antibody ( $\mathrm{h}$-EGF-R). A431 cells overexpressing the human endogenous EGF-R (h-EGF-R, $170 \mathrm{kDa}$ ) was used as positive control.

Plasma creatinine, urea, and electrolyte concentrations were determined with a Monarch multiparametric autoanalyzer (Instrumentation Laboratory, Paris, France).

Tubular activities. The $5^{\prime}$-nucleotidase $\left(5^{\prime}\right.$-Nuc), $\gamma$-glutamyl transpeptidase $(\boldsymbol{\gamma}-\mathrm{GT})$ and $\mathrm{Na}-\mathrm{K}$-ATPase activities were determined as described previously (37).

Expression of data and statistical analysis. Data are expressed as means plus or minus SEM. Differences between the experimental groups were evaluated using one-way ANOVA, which was followed, when significant, by a Bonferroni test.

\section{Results}

Generation of $\gamma G T-E G F-R-M$ transgenic mice. Transgenic mice expressing EGF-R-M under the control of type-1 $\gamma \mathrm{GT}$ promoter were generated by microinjecting onecell C57BL $/ 6 \times$ DAB2 mouse zygotes with the transgene construct. Three founders (Fo-8, Fo-20, and Fo35) were identified by Southern blot analysis of tail DNA. BamH1 digestion of genomic DNA from transgenic mice released the predicted $5.0-\mathrm{kb}$ transgene, which was integrated in multiple copies in a head-totail array, as detected by hybridization with the transgene-specific human $\beta$-globin poly $(\mathrm{A})$ probe. Line 20, expressing the highest level of the transgene, was selected for further analysis.

Tissue-specific expression of $\gamma G T-E G F-R-M$ transgene. Expression of the transgene was investigated by Northern blot analysis of RNA from transgenic mouse tissues using the transgene-specific human $\beta$-globin poly(A) probe. The $3.0-\mathrm{kb}$ transcript was of the predicted size for the transgene and was detected in kidney, but not in liver, spleen, heart, testis, brain, and gut (Figure 1a).

The distribution of EGF-R-M protein was evaluated in kidneys from transgenic and wild-type animals by immunohistochemistry using a monoclonal antihuman EGF-R antibody, which is specific for the human receptor and does not cross-react with that of the mouse. As expected, EGF-R-M immunoreactivity could not be revealed in any structure of kidneys from wild-type animals (Figure 1b, left panel). In contrast, EGF-R-M protein was detected in proximal tubules of transgenic mice (Figure 1b, middle panel). The staining was restricted to basolateral cellular membranes and was more intense in the tubules localized into the deep cortex (Figure 1b, right panel). No specific staining was observed in glomeruli, vessels, and distal tubules. In proximal tubular cells from transgenic, but not from wild-type mice, a 110-kDa protein was detected by Western blot with a specific anti-human EGF-R antibody corresponding to the transgene (Figure $1 c$, lower panel). Using a specific anti-EGF-R intracellular domain antibody, Western blot analysis showed that the amount of endogenous EGF-R was similar in cells from transgenic and wild-type animals (Figure 1c, upper panel).

Inbibition of EGF-R function. We next analyzed EGF-R autophosphorylation in kidneys from wild-type and transgenic mice, 10 and 20 minutes after intracardiac 
Table 1

Tubular function in wild-type and transgenic mice

\begin{tabular}{|c|c|c|c|c|c|c|c|c|c|}
\hline & \multicolumn{6}{|c|}{ Urinary solute excretions } & \multicolumn{3}{|c|}{ Enzymatic activities } \\
\hline & $\mathrm{Na} / \mathrm{Cr}$ & $\mathrm{K} / \mathrm{Cr}$ & $\mathrm{Cl} / \mathrm{Cr}$ & $\mathrm{Ca} / \mathrm{Cr}$ & $\mathrm{P} / \mathrm{Cr}$ & $\mathrm{G} / \mathrm{Cr}$ & $\gamma$-GT & $5^{\prime}$-Nuc & Na-K-ATPase \\
\hline WT & $93 \pm 16$ & $49 \pm 5$ & $90 \pm 12$ & $0.45 \pm 0.03$ & $23 \pm 4$ & $0.36 \pm 0.11$ & $0.55 \pm 0.02$ & $7.5 \pm 0.8$ & $31 \pm 1$ \\
\hline EGF-R-M & $102 \pm 12$ & $52 \pm 2$ & $94 \pm 10$ & $0.44 \pm 0.07$ & $27 \pm 5$ & $0.37 \pm 0.19$ & $0.58 \pm 0.05$ & $8.1 \pm 0.3$ & $31 \pm 2$ \\
\hline
\end{tabular}

Data are means \pm SEM. No significant difference appeared, for any parameters between transgenic and wild-type mice. WT, wild-type mice; EGF-R-M, transgenic mice; $\mathrm{Cr}$, creatinine; G, glucose; $\gamma$-GT, $\gamma$-glutamyl transpeptidase (U/mg protein); $5^{\prime}$-Nuc, $5^{\prime}$-nucleotidase (nmol/min $/ \mathrm{mg}$ protein); Na-K-ATPase (nmol/min/mg protein).

injection of EGF. In wild-type mice, EGF injection resulted in phosphorylation of renal EGF-R, corresponding to the 170-kDa band (Figure 2a). The effect was more intense at 10 minutes than at 20 minutes. In transgenic mice, EGF-induced phosphorylation of endogenous EGF-R was strongly reduced compared with wild-type animals at any time point (Figure 2 , a and $b$ ).

Since a previous work (38) reported that transfection of the EGF-R-M in Chinese hamster ovary (CHO) cells abolished the high-affinity state of the wild-type receptor, we analyzed the binding of ${ }^{125}$ I-EGF in proximal tubules isolated from wild-type and transgenic animals. Scatchard analysis of the binding data (Figure 2c) indicated that in wild-type tubules EGF-R exhibited two classes of binding sites: approximately $10 \%$ of the sites $(8.5 \pm 0.2 \mathrm{fmol} / \mathrm{mg}$ protein) exhibited a high affinity for the ligand with a $K_{d}$ value of $0.4 \times 10^{-9} \mathrm{M}$, the remaining binding sites ( $83 \pm 2.3 \mathrm{fmol} / \mathrm{mg}$ protein) exhibited a low affinity with a $K_{d}$ value of $3.1 \times 10^{-9} \mathrm{M}$. In proximal tubules from transgenic mice, the proportion of highaffinity binding sites was reduced, whereas the number of low-affinity binding sites $\left(K_{d}\right.$ value of $\left.3 \times 10^{-9} \mathrm{M}\right)$ was markedly increased $(267 \pm 4.4, P<0.001)$.
Consequences of $\gamma G T-E G F-R-M$ transgene expression on renal morphology and function. In transgenic mice, renal glomerular and tubular morphology was normal (Figure $3 b$ ), comparable under basal conditions to that of wild-type animals (Figure 3a). Plasma creatinine, urea, sodium, potassium, chloride, calcium, and phosphorus concentrations, evaluated between 1 and 18 months after birth, were comparable in transgenic and wild-type animals at any time point (data not shown). Creatinine clearance was similar in the two experimental groups and was around $0.12 \mathrm{~mL} / \mathrm{min}$ at 12 months after birth.

Since it has been reported that EGF can modulate tubular function (39), we next investigated the impact of transgene expression on transport, enzymatic, and pump tubular functions. Expression of EGF-R-M did not affect the ability of proximal tubules to reabsorb solutes (Table 1). Similarly, the activities of $5^{\prime}$-nucleotidase and of $\gamma$-GT, two enzymes of proximal tubular brush-border membranes, as well as that of $\mathrm{Na}-\mathrm{K}$ ATPase, a pump of basolateral membranes, were comparable in transgenic and wild-type mice (Table 1).

Progression of renal lesions was reduced in EGF-R-M transgenic mice. The next step was to evaluate the conse-
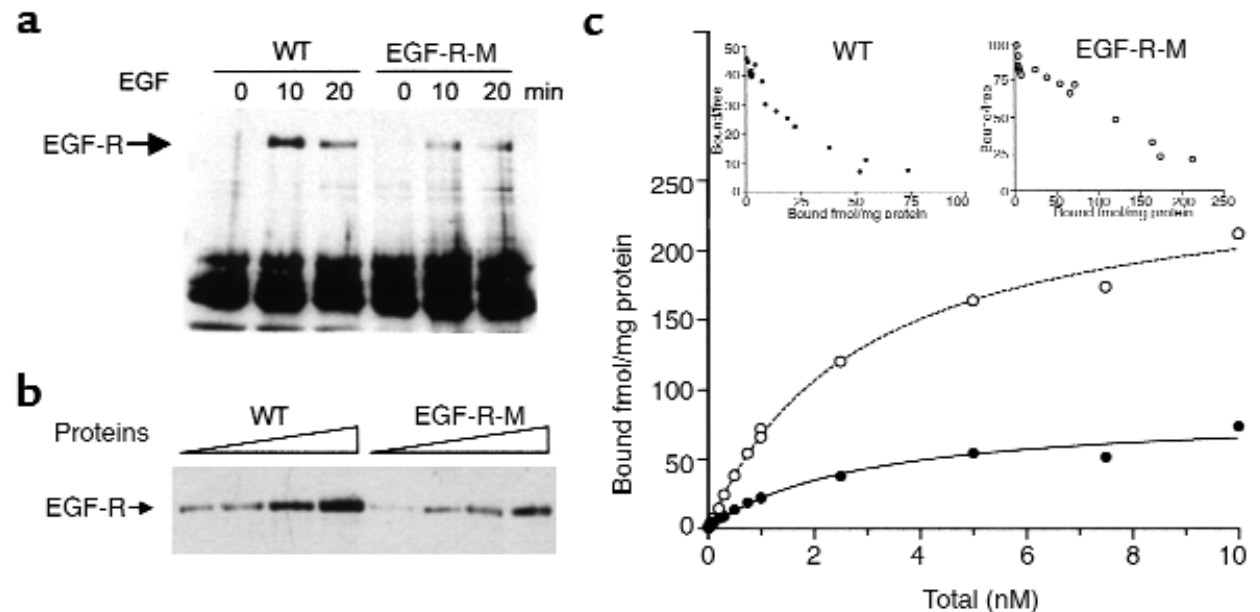

\section{Figure 2}

Transgene function. (a and $\mathbf{b})$ Western blot analysis of EGF-R autophosphorylation in transgenic (EGF-R-M) and wild-type (WT) mice. (a) Transgene expression reduced the phosphorylation of EGF-R in transgenic mice after EGF injection. Proteins were extracted from whole kidney and immunoprecipitated with an anti-EGF-R antibody. The immunoprecipitate was probed with the RC-20 anti-phosphotyrosine antibody. (b) Increasing amounts $(50,100,200$, and $300 \mu \mathrm{g}$ ) of proteins were immunoprecipitated to validate the method used to quantify EGF-R phosphorylation (linear regression: $r^{2}=1.00$ and 0.985 for wild-type and transgenic mice, respectively). (c) Binding curves and Scatchard plots (inset) of ${ }^{125} \mathrm{I}$-EGF binding. Transgene expression abolished the high-affinity state of the endogenous receptor in isolated proximal tubules. Note that different scales were used in the two insets. 
Figure 3

Morphology of sham-operated ( $\mathbf{a}$ and $\mathbf{b}$ ) and remnant kidneys ( $\mathbf{c}$ and $\mathbf{d}$ ) from wild-type ( $\mathbf{a}$ and c) and transgenic (b and $\mathbf{d}$ ) mice, 8 months after surgery. Nephron reduction induced several morphological changes of proximal tubules (compare $\mathbf{c}$ with $\mathbf{a}$ and $\mathbf{b}$ ), which were prevented by transgene expression (compare $\mathbf{d}$ with $\mathbf{c}$ ). PAS staining, $\times 200$.

quences of inhibition of EGF pathway in the development of renal lesions in two models of renal injury: subtotal nephrectomy and prolonged renal ischemia.

Subtotal nephrectomy $(N x)$. In wildtype animals, $\mathrm{Nx}$ resulted in marked tubular alterations, which were apparent 8 months after surgery (Figure 3c). At that time, tubules showed protein reabsorption droplets, proteinaceous casts, and focal dilations. Proximal tubular epithelial cells appeared hypertrophied with a reduced nuclear/cytoplasmic ratio and a loss of microvilli. Glomeruli were hypertrophied and showed an increased amount of PAS-positive material. These changes were blunted in transgenic mice: despite nephron reduction, the size of tubular cells appeared normal, their microvilli were preserved, and no tubular dilations were observed (Figure 3d). To quantify the differences between the two groups of mice, we scored the damaged tubules: EGF-R-M expression significantly reduced the score of tubular dilation $(0.56 \pm$ 0.16 vs. $1.15 \pm 0.15, P<0.05$ ).

Since EGF is a potent mitogen for renal tubular cells $(6,7)$ and a relationship between cell proliferation and tubular lesions has been suggested after nephron reduction (40), PCNA immunostaining was performed on kidney sections from transgenic and wild-type animals. In wild-type mice, the number of tubular PCNA-positive cells was slightly higher in remnant kidneys of nephrectomized mice, as compared with shamoperated animals, 8 months after surgery (Table 2). The number of proliferating cells was fewer, but not statistically different, in remnant kidneys of transgenic mice than in those of wild-type mice. Since these results contrasted with the morphological aspect of tubules and the

\footnotetext{
Figure 4

Morphology of control ( $\mathbf{a}$ and $\mathbf{b}$ ) and postischemic kidneys ( $\mathbf{c}$ and $\mathbf{d}$ ) from wild-type ( $\mathbf{a}$ and $\mathbf{c}$ ) and transgenic (b and $\mathbf{d}$ ) mice, 28 days after ischemia. Prolonged renal ischemia induced tubular atrophy, interstitial fibrosis, and mononuclear cell infiltrates (compare $\mathbf{c}$ to $\mathbf{a}$ and b), which were reduced by transgene expression (compare $\mathbf{d}$ to c). Masson's trichrome staining, $\times 200$.
}
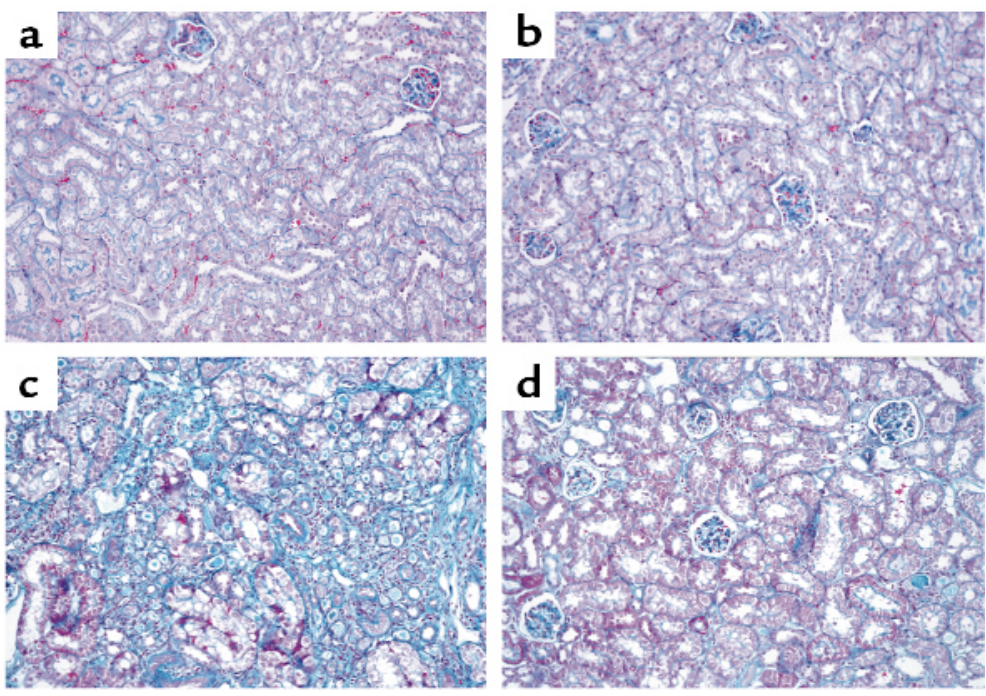

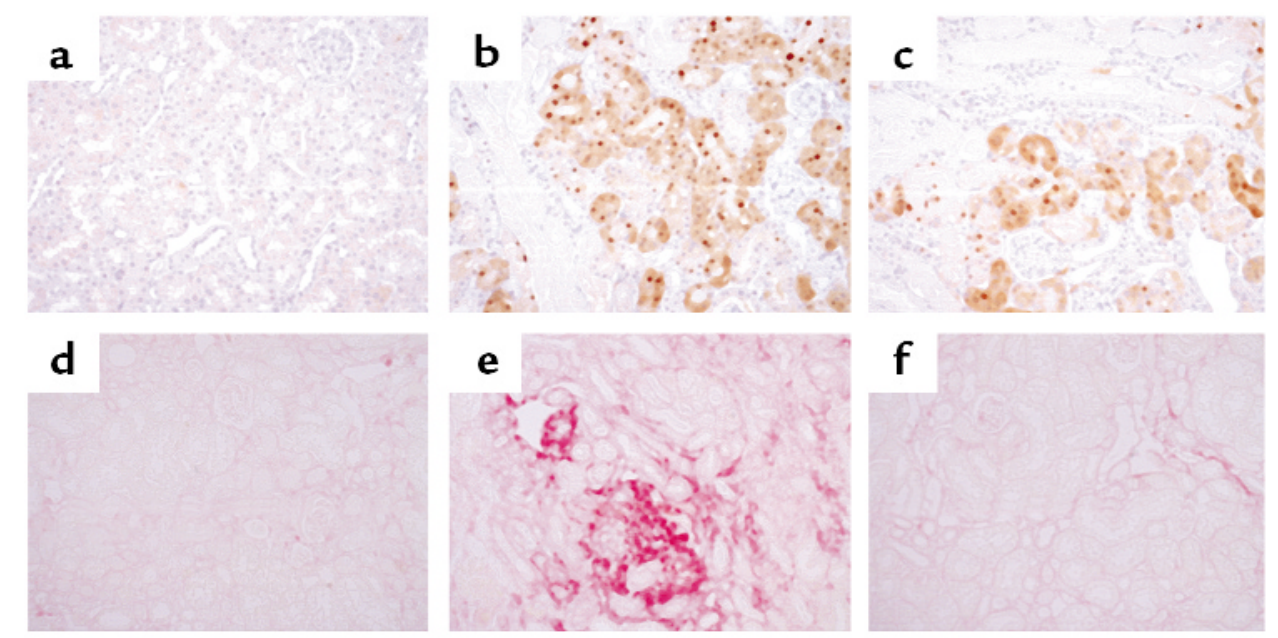

\section{Figure 5}

Cell proliferation and collagen accumulation in control ( $\mathbf{a}$ and $\mathbf{d}$ ) and postischemic kidneys (b, $\mathbf{c}, \mathbf{e}, \mathbf{f})$ from wild-type (a, b, $\mathbf{d}, \mathbf{e})$ and transgenic (c and $\mathbf{f}$ ) mice. (a-c) PCNA immunostaining, 2 days after PRI. PRI induced proliferation of tubular cells (compare $\mathbf{b}$ with $\mathbf{a}$ ), which was markedly reduced by transgene expression (compare $\mathbf{c}$ with $\mathbf{b}$ ). (d-f) Sirius red staining, 28 days after ischemia. PRI induced interstitial collagen accumulation (compare $\mathbf{e}$ with $\mathbf{d}$ ), which was markedly reduced by transgene expression (compare $\mathbf{f}$ with $\mathbf{e}) . \times 200$.

necrosis of tubular cells after 50 minutes of ischemia, but reduced dramatically the development of renal lesions thereafter. Indeed, 28 days after surgery, in transgenic postischemic kidneys, the majority of tubules were normal, whereas only few areas of interstitial fibrosis and mononuclear cell infiltrates appeared (Figure $4 d$ ). To quantify the beneficial effect of transgene expression, we scored the areas with tubulo-interstitial lesions: the score of damaged areas was reduced in transgenic postischemic kidneys, as compared with wild-type kidneys $(1.63 \pm 0.37$ vs. $2.77 \pm 0.15, P<0.05)$.

To gain further insights into the mechanisms by which a dominant-negative mutant of EGF- $\mathrm{R}$ protects from the development of renal lesions after PRI, we evaluated the impact of transgene expression on cellular proliferation rate and on collagen deposition. In wild-type animals, the number of PCNA-labeled cells increased significantly after ischemia (Figure 5b). Proliferative activity concerned mainly proximal tubules. As shown in Figure 6 , the increment was maximal at day 2 and remained higher than in wild-type nonoperated kidney at day 28 after surgery. In transgenic mice, the number of tubular PCNA-labeled cells was significantly reduced 2 and 4 days after surgery (Figure 5c and Figure 6).

Collagen deposition was evaluated on postischemic kidneys using Sirius red staining, a histological method currently used to detect this protein. In wild-type animals, 28 days after ischemia marked collagen deposition was detected in interstitium surrounding the proximal tubules and, to a lesser extent, within glomeruli (Figure 5e). This phenomenon was reduced by transgene expression (Figure 5f). Quantification of collagen deposition, performed as described in Methods, showed that 28 days after ischemia, the score of redstained areas was 1.93 plus or minus 0.1 in wild-type animals, whereas it reached only 0.99 plus or minus 0.1 in transgenic mice $(P<0.005)$.

\section{Discussion}

Previous works suggested that activation of EGF-R could play a major role in the evolution of renal injury $(1,2)$. In the present study, we established a line of transgenic mice that selectively expressed in the kidney a dominant-negative mutant of EGF-R under the control of the $\gamma$ GT-1 promoter. We demonstrated that transgenic mice, which had normal renal morphology and renal function under basal conditions, became resistant to the development of renal lesions after renal injury. This effect was associated with a reduction of tubular cellular proliferation, interstitial deposition of extracellular matrix, and mononuclear cell infiltration. Since in

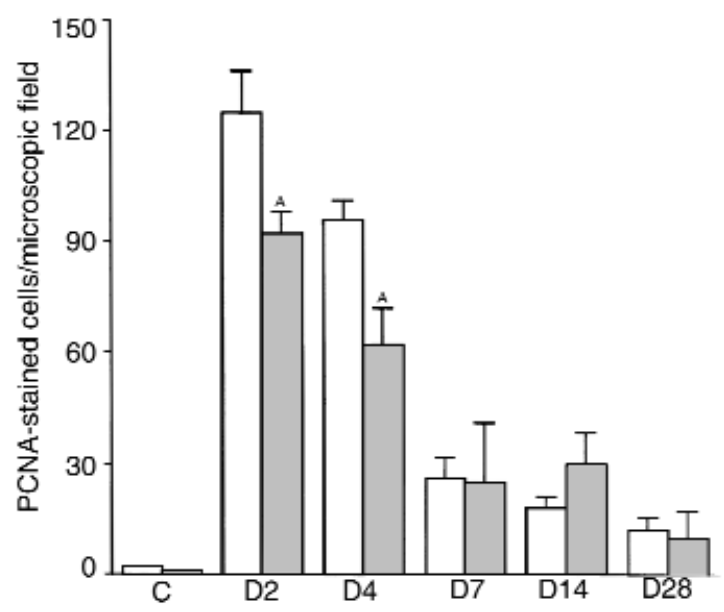

\section{Figure 6}

Tubular cell proliferation in control (C) and postischemic kidneys from wild-type (open bars) and transgenic (gray bars) mice, 2, 4, 7, 14, and 28 days after ischemia. The number of PCNA-labeled cells, which markedly increased after ischemia, was reduced by transgene expression. Data are means \pm SEM. ${ }^{A} A N O V A$, transgenic versus wild-type mice; $P<0.05$. 
Table 2

Cell proliferation in kidneys from wild-type and transgenic mice 8 months after surgery

$$
\begin{aligned}
& \text { PCNA-stained nuclei/ } \\
& \text { microscopic field }
\end{aligned} \quad \begin{gathered}
\text { Hematoxylin-stained } \\
\text { nuclei/tubular section }
\end{gathered}
$$

WT

$\begin{array}{lcc}\text { Sham-operated } & 1.98 \pm 0.23 & 6.25 \pm 0.38 \\ \text { Nephrectomized } & 5.47 \pm 2.98 & 19.60 \pm 2.15^{\mathrm{A}} \\ \text { EGF-R-M } & & \\ \text { Sham-operated } & 3.20 \pm 0.29 & 5.88 \pm 0.23 \\ \text { Nephrectomized } & 3.02 \pm 0.52 & 12.94 \pm 1.50^{\mathrm{A}, \mathrm{B}} \\ \end{array}$

Data are means \pm SEM. ${ }^{A}$ Nephrectomized vs. sham-operated mice, $P<0.005$ using ANOVA. ${ }^{\text {BT }}$ ransgenic vs. wild-type mice, $P<0.05$ using ANOVA.

our model the truncated EGF-R is expressed exclusively in proximal tubular cells, these results provide evidence for a cross-talk between tubular and interstitial cells in injured kidneys and demonstrate that tubular EGF-R activation is a pivotal factor in this interaction.

HERCD-533, the truncated EGF-R used as a dominant-negative mutant in the present study, lacks most of cytoplasmic domain, including the tyrosine kinase domain (38). Previous studies $(38,42,43)$ have shown that transfection of this mutant in various cell types inhibits receptor autophosphorylation, reduces the receptor endocytosis and degradation, and diminishes its mitogenic and oncogenic signals. This mutant was also reported to be successfully expressed in vivo in the skin, under the control of keratin promoter, and to behave as an efficient dominant-negative mutant (44). As expected, in the present study targeted renal expression of the HERCD-533 mutant reduced the phosphorylation of the endogenous EGF-R in the kidney upon infusion of EGF. In our study, that expression of the transgene in proximal tubules reduced the percentage of high-affinity binding sites and increased dramatically the density of low-affinity receptors was accounted for by the fact that the transgene was overexpressed versus the wild-type endogenous receptor (Figure 1 and 2 ) and behaved as a dominant-negative mutant. Our data are in agreement with previous in vitro studies (38, 43) that reported a similar effect when cells lacking endogenous EGF-R were cotransfected with both wildtype and mutant receptors. Our work evidenced that this phenomenon occurs in vivo and may therefore be instrumental in modulation of EGF pathway.

The observation that phosphorylation of endogenous receptor was undetectable in both wild-type and transgenic mice under basal conditions could explain the normal renal phenotype, in terms of morphology and function, in nonoperated transgenic mice. Nevertheless, these results argue against the idea that EGF-R signaling plays a major role in modulation of tubular solute transport under physiological conditions (45).

In our study, the selective expression of the transgene in renal proximal tubules did not affect the develop- ment of the kidney. These findings apparently contrast with the observation that EGF-R inactivation by homologous recombination resulted in three different phenotypes, ranging from peri-implantation lethality to postnatal lethality, or in defects of all organs undergoing branching morphogenesis, including kidney (27-29). However, it should be noted that: (a) the promoter used in the current study is weakly expressed during the development (31) and (b) the phosphorylation of endogenous receptor was not totally prevented in a dominant-negative strategy, as shown by Figures 2 , $\mathrm{a}$ and $\mathrm{b}$, and by previous works $(38,42-44)$ using the same strategy. It is noteworthy that also in waved-2 mutant mice, in which the EGF-R kinase activity is only partially impaired, kidneys developed normally (30).

Because EGF was suspected to play a role in several renal diseases $(1,2)$, we investigated in the present study whether functional EGF-R inactivation in proximal tubular cells could protect from the progression of renal lesions after an initial injury. Since high expression levels of the transgene are required to reveal the dominantnegative effect of the mutated EGF-R, renal injury experiments were performed only on mice of line 20 , which expressed the highest levels of the transgene. Indeed, homozygous mice of lines 8 and 35 expressed the transgene at a lower level than heterozygous mice of line 20, which were only partially protected against renal alterations after renal injury (data not shown). That renal morphology and function was normal under basal conditions in all three lines of transgenic mice argues against an ectopic effect of the transgene in line 20. Furthermore, it must be pointed out that all the data obtained in this line favor a specific effect of the transgene.

That, after nephron reduction, tubular dilations and proximal cell proliferation were significantly reduced in transgenic mice demonstrated that activation of EGF-R pathway is at least one of the first events triggering cell proliferation after nephron reduction. These results are consistent with the observation that the decrease of EGF-R phosphorylation in mice carrying both the orpk cystic mutation (in which a point mutation induces renal cysts, tubular hyperplasia, and increased EGF-R activity) (46) and the waved-2 mutation (in which a point mutation decreases EGF-R tyrosine kinase activity) (30) diminished cell proliferation and collecting tubular cyst formation in double-mutant mice (25).

Regarding the natural history of renal mass reduction in mice, it should be noted that, at variance with rats $(47,48)$, wild-type mice developed only moderate tubular lesions after reduction of $75 \%$ of total renal mass, and this occurred exclusively from 8 months after surgery. This discrepancy may be accounted for by the genetic background of the mice used. Indeed, recent works suggested that genetic factors influence the response to nephron reduction. Esposito et al. (49) have shown recently that unilateral nephrectomy induced glomerulosclerosis in ROP, but not in C57BL/6 mice, whereas Kren and Hostetter (50) have reported that C57BL/ 6 mice were resistant to the devel- 
opment of renal lesions after subtotal nephrectomy, at least until 44 weeks after nephron reduction. The mice used in the present work are generated by intercrossing $\mathrm{C} 57 \mathrm{BL} / 6 \times \mathrm{DAB} 2$ mice, which confirms the low susceptibility to renal deterioration of C57BL/6 background and shows that this phenotype is maintained in a outbred strain.

PRI, another model of renal injury, mimics the evolution and characteristics of renal lesions observed in human renal diseases, such as renal venous thrombosis, hemolytic uremic syndrome, or kidney transplants complicated by renal arterial stenosis. That EGF-R-M transgenic mice were also protected against this injury stresses the major involvement of EGF in renal pathology. These results apparently contrast with the beneficial effect of EGF infusion reported after short-term renal ischemic injury by Humes et al. (12). It should be stressed, however, that our model differs from shortterm renal ischemia model, since, in the latter, the kidney structure is restored ad integrum and EGF affects primarily the rate of recovery, a phenomenon that was not investigated in the present study.

It is noteworthy that the beneficial effect of the transgene concerns not only a decrement of tubular cell proliferation, but also a reduction of interstitial fibrosis and mononuclear cell infiltration. Involvement of EGF pathway in fibrogenetic processes has been reported previously in the lung. Indeed, using a transgenic approach, Korfhogen et al. (51) showed that mice that overexpressed TGF- $\alpha$ under the control of surfactant protein $\mathrm{C}$ (SP-C) promoter developed fibrotic lesions in several pulmonary regions surrounding the epithelial cells that expressed the transgene. These lesions were reversed in double-transgenic mice in which the EGF signaling was blocked by breeding the SP-C-TGF- $\alpha$ mice with either the SP-C-EGF-R-M mice (a line of mice which selectively expressed in lung the same EGF-R mutant used in the present work) or the waved-2 mice (52).

The molecular and cellular mechanisms by which inhibition of EGF pathway in proximal tubules prevented interstitial fibrosis remain to be elucidated. A recent work (53) suggests that, under pathological conditions, conversion from proximal tubular cells to fibroblasts occurs. Fsp-1 (fibroblast-specific protein 1), a gene encoding a fibroblast-specific protein, would be the conversion driver. In a mouse model of anti-basement membrane disease, tubular epithelial cells have been reported to express FSP-1 and to be converted into a fibroblast phenotype in the areas of fibrogenesis (53). Since addition of EGF to proximal tubular cells, in vitro, strongly induced FSP-1 expression and mesenchymal cell transformation and synergized with TGF- $\beta$ to increase extracellular matrix production (54), we speculate that activation of EGF pathway in proximal tubules after renal injury could be the first event that triggers the epithelialfibroblast conversion and, ultimately, renal fibrosis. Alternatively, it is conceivable that EGF affects directly the capacity of tubular cells to synthesize matrix collagens under pathological conditions (55) inasmuch as we observed a reduction of collagen accumulation in the injured kidneys of EGF-R-M transgenic mice. Finally, since EGF could modulate the production of tubular cytokines $(56,57)$, these mediators might participate in the cross-talk between tubular and interstitial cells.

In conclusion, using a dominant-negative transgenic approach, we showed that targeted inhibition of EGF signaling in the kidney successfully prevents the development of renal lesions after renal injury. Previous transgenic models have evidenced that overexpression of growth factors in the kidney induced the destruction of both glomerular and tubular structures (18), arguing in favor of a key involvement of these paracrine/autocrine molecules in renal deterioration process. The present findings provide the first evidence, to our knowledge, that strategies aiming to block growth-factor pathways could be useful in delaying the progression of chronic renal failure and the occurrence of end-stage renal failure in humans.

\section{Acknowledgments}

We are deeply grateful to Uriel Hazan for assistance in plasmid construction, Marie-Claire Gubler and Patrick Bruneval for histological advice, and Marco Pontoglio and Marie-Edith Oblim for critical advice. We thank Michael W. Lieberman for $\gamma$-GT plasmid, Axel Ullrich for HERCD-533 plasmid and mAb108 antibody, and Eva Earp for Ab-22 antibody. This work was supported in part by a Cégetel Company grant fellowship to M. Hekmati and by grants from INSERM, Université René Descartes and Denis Diderot, Laboratoire de Recherches Physiologiques, and Association pour la Recherche sur le Cancer.

\footnotetext{
1. Wolf, G., and Neilson, E.G. 1991. Molecular mechanisms of tubulointerstitial hypertrophy and hyperplasia. Kidney Int. 39:401-420.

2.Hamm, L.L., Hering, S.K., and Vehaskari, V.M. 1993. Epidermal growth factor and the kidney. Semin. Nephrol. 13:109-115.

3. Moghal, N., and Sternberg, P.W. 1999. Multiple positive and negative regulators of signaling by the EGF-receptor. Curr. Opin. Cell. Biol. 11:190-196. 4. Salido, E.C., Lakshmanan, J., Fisher, D.A., Shapiro, L.J., and Barajas, L. 1991. Expression of epidermal growth factor in the rat kidney. An immunocytochemical and in situ hybridization study. Histochemistry. 96:65-72.

5. Breyer, M.D., Redha, R., and Breyer, J.A. 1990. Segmental distribution of epidermal growth factor binding sites in rabbit nephron. Am. J. Physiol. 259:F553-F558.

6. Norman, J., et al. 1987. EGF-induced mitogenesis in proximal tubular cells: potentiation by angiotensin II. Am. J. Physiol. 253:F299-F309.

7. Goodyer, P.R., Kachra, Z., Bell, C., and Rozen, R. 1988. Renal tubular cells are potential targets for epidermal growth factor. Am. J. Physiol. 255:F1191-F1196.

8. Sponsel, H.T., Breckon, R., Hammond, W., and Anderson, R.J. 1994. Mechanisms of recovery from mechanical injury of renal tubular epithelial cells. Am. J. Physiol. 267:F257-F264.

9. Kjelsberg, C., et al. 1997. Met-/- kidneys express epithelial cells that chemotax and form tubules in response to EGF receptor ligands. Am.J. Physiol. 272:F222-F228.

10. Creely, J.J., DiMari, S.J., Howe, A.M., Hyde, C.P., and Haralson, M.A. 1990. Effects of epidermal growth factor on collagen synthesis by an epithelioid cell line derived from normal rat kidney. Am. J. Pathol. 136:1247-1257.

11. Taub, M., Wang, Y., Szczesny, T.M., and Kleinman, H.K. 1990. Epidermal growth factor or transforming growth factor alpha is required for kidney tubulogenesis in matrigel cultures in serum-free medium. Proc. Natl. Acad. Sci. USA. 87:4002-4006.

12. Humes, H.D., Cieslinski, D.A., Coimbra, T.M., Messana, J.M., and Galvao, C. 1989. Epidermal growth factor enhances renal tubule cell regeneration and repair and accelerates the recovery of renal function in postischemic acute renal failure. J. Clin. Invest. 84:1757-1761.
} 
13. Coimbra, T.M., Cieslinski, D.A., and Humes, H.D. 1990. Epidermal growth factor accelerates renal repair in mercuric chloride nephrotoxicity. Am. J. Physiol. 259:F438-F443.

14. Jennische, E., Andersson, G., and Hansson, H.A. 1987. Epidermal growth factor is expressed by cells in the distal tubules during postnephrectomy renal growth. Acta Physiol. Scand. 129:449-450.

15. Kanda, S., et al. 1991. Transient increase in renal epidermal growth factor content after unilateral nephrectomy in the mouse. Acta. Endocrinol. 124:188-193.

16. Miller, S.B., Rogers, S.A., Estes, C.E., and Hammerman, M.R. 1992. Increased distal nephron EGF content and altered distribution of peptide in compensatory renal hypertrophy. Am. J. Physiol. 262:F1032-F1038.

17. Muchaneta-Kubara, E.C., Sayed-Ahmed, N., and el Nahas, A.M. 1995. Subtotal nephrectomy: a mosaic of growth factors. Nephrol. Dial. Transplant. 10:320-327.

18. Terzi, F., Burtin, M., and Friedlander, G. 1998. Early molecular mechanisms in the progression of renal failure: role of growth factors and protooncogenes. Kidney Int. Suppl. 53:S68-S73.

19. Wilson, P.D., Du, J., and Norman, J.T. 1993. Autocrine, endocrine and paracrine regulation of growth abnormalities in autosomal dominant polycystic kidney disease. Eur. J.Cell Biol. 61:131-138.

20. Orellana, S.A., Sweeney, W.E., Neff, C.D., and Avner, E.D. 1995. Epidermal growth factor receptor expression is abnormal in murine polycystic kidney. Kidney Int. 47:490-499.

21. Uhlman, D.L., et al. 1995. Epidermal growth factor receptor and transforming growth factor alpha expression in papillary and nonpapillary renal cell carcinoma: correlation with metastatic behavior and prognosis. Clin. Cancer Res. 1:913-920.

22. Lowden, D.A., et al. 1994. Renal cysts in transgenic mice expressing transforming growth factor-alpha. J. Lab. Clin. Med. 124:386-394.

23. Stocklin, E., Botteri, F., and Groner, B. 1993. An activated allele of the cerbB-2 oncogene impairs kidney and lung function and causes early death of transgenic mice. J.Cell Biol. 122:199-208.

24. Schaffner, D.L., et al. 1993. Targeting of the rasT24 oncogene to the proximal convoluted tubules in transgenic mice results in hyperplasia and polycystic kidneys. Am. J. Pathol. 142:1051-1060.

25. Richards, W.G., et al. 1998. Epidermal growth factor receptor activity mediates renal cyst formation in polycystic kidney disease. J. Clin. Invest. 101:935-939.

26. Prewett, M., et al. 1998. Mouse-human chimeric anti-epidermal growth factor receptor antibody C225 inhibits the growth of human renal cell carcinoma xenografts in nude mice. Clin. Cancer Res. 4:2957-2966.

27. Miettinen, P.J., et al. 1995. Epithelial immaturity and multiorgan failure in mice lacking epidermal growth factor receptor. Nature. 376:337-341.

28. Sibilia, M., and Wagner, E.F. 1995. Strain-dependent epithelial defects in mice lacking the EGF receptor. Science. 269:234-238.

29. Threadgill, D.W., et al. 1995. Targeted disruption of mouse EGF receptor: effect of genetic background on mutant phenotype. Science. 269:230-234

30. Luetteke, N.C., et al. 1994. The mouse waved-2 phenotype results from a point mutation in the EGF receptor tyrosine kinase. Genes Dev. 8:399-413.

31. Jacquemin, E., et al. 1990. Pattern of expression of gamma-glutamyl transpeptidase in rat liver and kidney during development: study by immunochemistry and in situ hybridization. J. Pediatr. Gastroenterol. Nutr. 11:89-95.

32. Terzi, F., et al. 1997. Reduction of renal mass is lethal in mice lacking vimentin. Role of endothelin-nitric oxide balance. J. Clin. Invest. 100:1520-1528

33. Terzi, F., Beaufils, H., Laouari, D., Burtin, M., and Kleinknecht, C. 1992. Renal effect of anti-hypertensive drugs depends on sodium diet in the excision remnant kidney model. Kidney Int. 42:354-363.

34. Terzi, F., et al. 1997. Normal tubular regeneration and differentiation of the post-ischemic kidney in mice lacking vimentin. Am. J. Pathol. 150:1361-1371.
35. Iimura, O., Vrtovsnik, F., Terzi, F. and Friedlander, G. 1997. HMG-CoA reductase inhibitors induce apoptosis in mouse proximal tubular cells in primary culture. Kidney Int. 52:962-972.

36. Raij, L., Azar, S., and Keane, W. 1984. Mesangial immune injury, hypertension, and progressive glomerular damage in Dahl rats. Kidney Int. 26:137-143

37. Laouari, D., et al. 1997. Subtotal nephrectomy alters tubular function: effect of phosphorus restriction. Kidney Int. 52:1550-1560.

38. Livneh, E., et al. 1986. Reconstitution of human epidermal growth factor receptors and its deletion mutants in cultured hamster cells. J. Biol. Chem. 261:12490-12497.

39. Harris, R.C. 1991. Potential physiologic roles for epidermal growth factor in the kidney. Am. J. Kidney Dis. 17:627-630.

40. Terzi, F., et al. 2000. Sodium restriction decreases AP-1 activation after nephron reduction in the rat: role in the progression of renal lesions. Exp. Nephrol. 8:104-114.

41. Terzi, F., et al. 1995. Subtotal but not unilateral nephrectomy induces hyperplasia and protooncogene expression. Am. J. Physiol. 268:F793-F801.

42. Kashles, O., Yarden, Y., Fischer, R., Ullrich, A., and Schlessinger, J. 1991. A dominant negative mutation suppresses the function of normal epidermal growth factor receptors by heterodimerization. Mol. Cell. Biol. 11:1454-1463.

43. Redemann, N., et al. 1992. Anti-oncogenic activity of signalling-defective epidermal growth factor receptor mutants. Mol. Cell Biol. 12:491-498.

44. Murillas, R., et al. 1995. Expression of a dominant negative mutant of epidermal growth factor receptor in the epidermis of transgenic mice elicits striking alterations in hair follicle development and skin structure. EMBO J. 14:5216-5223

45. Harris, R.C. 1991. Potential physiologic roles for epidermal growth factor in the kidney. Am. J. Kidney Dis. 17:627-630.

46. Moyer, J.H., et al. 1994. Candidate gene associated with a mutation causing recessive polycystic kidney disease in mice. Science. 264:1329-1333.

47. Morrison, A.B. 1962. Experimentally induced chronic renal insufficiency in the rat. Lab. Invest. 11:321-332.

48. Shimamura, T., and Morrison, A.B. 1975. A progressive glomerulosclerosis occurring in partial five-sixths nephrectomized rats. Am. J. Pathol. 79:95-106.

49. Esposito, C., He, C.J., Striker, G.E., Zalups, R.K., and Striker, L.J. 1999. Nature and severity of the glomerular response to nephron reduction is strain-dependent in mice. Am. J. Pathol. 154:891-897.

50. Kren, S., and Hostetter, T.H. 1999. The course of the remnant kidney model in mice. Kidney Int. 56:333-337.

51. Korfhagen, T.R., et al. 1994. Respiratory epithelial cell expression of human transforming growth factor-alpha induces lung fibrosis in transgenic mice. J. Clin. Invest. 93:1691-1699.

52. Hardie, W.D., et al. 1996. Reversal of lung lesions in transgenic transforming growth factor alpha mice by expression of mutant epidermal growth factor receptor. Am. J. Respir. Cell Mol. Biol. 15:499-508.

53. Strutz, F., et al. 1995. Identification and characterization of a fibroblast marker: FSP1. J. Cell Biol. 130:393-405.

54. Okada, H., Danoff, T.M., Kalluri, R., and Neilson, E.G. 1997. Early role of Fsp1 in epithelial-mesenchymal transformation. Am. J. Physiol. 273:F563-574.

55. Orphanides, C., et al. 1997. Hypoxia stimulates proximal tubular cell matrix production via a TGF- $\beta_{1}$-independent mechanism. Kidney Int. 52:637-647.

56. Schena, F.P., Gesualdo, L., Grandaliano, G., and Montinaro, V. 1997. Progression of renal damage in human glomerulonephritides: is there sleight of hand in winning the game? Kidney Int. 52:1439-1457.

57. Malyankar, U.M., Almeida, M., Johnson, R.J., Pichler, R.H., and Giachelli, C.M. 1997. Osteopontin regulation in cultured rat renal epithelial cells. Kidney Int. 51:1766-1773. 